\title{
Assessment of ECH stray radiation levels at the W7-X Michelson Interferom- eter and Profile Reflectometer
}

\author{
Johan. W. Oosterbeek ${ }^{1, *}$, Neha Chaudhary ${ }^{1}$, Matthias Hirsch ${ }^{1}$, Udo Höfel ${ }^{1}$, Robert. C. Wolf ${ }^{1}$, and the W7-X team ${ }^{1}$ \\ ${ }^{1}$ Max-Planck-Institut für Plasmaphysik, Teilinstitut Greifswald, Wendelsteinstraße 1, 17491 Greifswald, Germany
}

\begin{abstract}
Electron Cyclotron Heating and Electron Cyclotron Current Drive are key components for heating and control in magnetically confined fusion plasmas. The high power microwaves are not always completely absorbed leading to stray radiation [1], [2]. At W7-X, the total injected microwave power can be up to $7.5 \mathrm{MW}$ @ $140 \mathrm{GHz}$ while the entire Electron Cyclotron Emission picked-up by an observer at the edge of the plasma is a fraction of a $\mathrm{mW}$. In the situation of a Michelson Interferometer, the principle measurement is the entire ECE spectrum. Thus, any stray radiation is bound to enter the spectrum. In this work initial stray radiation measurements without filters at the location of two microwave receivers - the Michelson Interferometer and the Profile Reflectometer - are discussed. The data is used to dimension a notch filter to be used with the broad band Michelson Interferometer.
\end{abstract}

\section{Introduction}

A Michelson Interferometer is being prepared for W7$\mathrm{X}$ operations from campaign OP1.2b onwards, scheduled July - October 2018. The objective of the work reported here is to find both the absolute power at the input of the instrument as well as the ratio of ECH to ECE power to assess the impact of stray radiation. In support of this, stray radiation measurements at the location of Michelson Interferometer as well as at the location of the Profile Reflectometer have been carried out during the previous W7-X experimental campaign (OP1.2a). Spurious gyrotron mode have not been included in this work.

\section{Instruments and waveguide system}

Both the Michelson Interferometer and the Profile Reflectometer use in-vessel horn antennae. The signals are relayed from the vessel by means of oversized waveguide to the Diagnostic Hall. A sketch is given in Fig. 1.

\subsection{Michelson Interferometer}

The Michelson Interferometer is designed to operate from $50 \cdots 300 \mathrm{GHz}$. The in-vessel antenna of the ECE diagnostic is located in module 4 (port 'AEE41'). It is constructed by a quasi-optic section of mirrors that project the collected beam onto a horn antenna with a diameter of 36 $\mathrm{mm}$. The output of the horn is tapered to $4 \mathrm{~mm}$ circular waveguide and subsequently passes a single disk mica vacuum window. On passing the window the waveguide is tapered to $28 \mathrm{~mm}$ smooth walled circular waveguide and split into X- and O-mode by a wire grid. These waveguides then continue to the diagnostic hall where, by means

*e-mail: hans.oosterbeek@ipp.mpg.de of switches, $\mathrm{X}$-mode is coupled to the radiometer and $\mathrm{O}$ mode to the Michelson Interferometer. The overall waveguide length between vessel and instrument is $\approx 22 \mathrm{~m}$. The losses of waveguide up to the radiometer have been measured in advance of OP1.2a using a $140 \mathrm{GHz}$ source and are $13.5+/-0.5 \mathrm{~dB}$ (waveguide relay in $\mathrm{TE}_{11}$ mode). The losses starting at the input of the wire grid through the vacuum windows, tapers and up to the horn antenna are estimated to be $3 \mathrm{~dB}$, resulting in an estimated overall transmission line loss of $16 \mathrm{~dB}$.

\subsection{Profile Reflectometer}

The edge Density Profile Reflectometer is situated in module 2 (port 'AEA21') and is still under development. In the case of the profile reflectometer the in-vessel antennas consists of two horns with diameter $62 \mathrm{~mm}$, located such that their beams intersect in the edge region of the plasma. The upper horn was used in the experiment. The horn guides the power into a section of circular waveguide that tapers to $4 \mathrm{~mm}$ diameter in order to pass the mica vacuum window. Following the window the waveguide is tapered up to $28 \mathrm{~mm}$ smooth walled circular waveguide and continues to the diagnostic hall. While the overall waveguide length is comparable to the ECE-system, the losses are lower. This can possibly be explained by fewer components in the Transmission Line (e.g. no X/O splitter, no polarisation tuner). The losses in the Tx-Line from window to instrument were measured, similarly as in Sec. 2.1, and are $8+/-0.5 \mathrm{~dB}$. The loss from vacuum window up to the in-vessel horn aperture is estimated at $2 \mathrm{~dB}$, such that the overall loss of the Tx-Line is set to $10 \mathrm{~dB}$. 


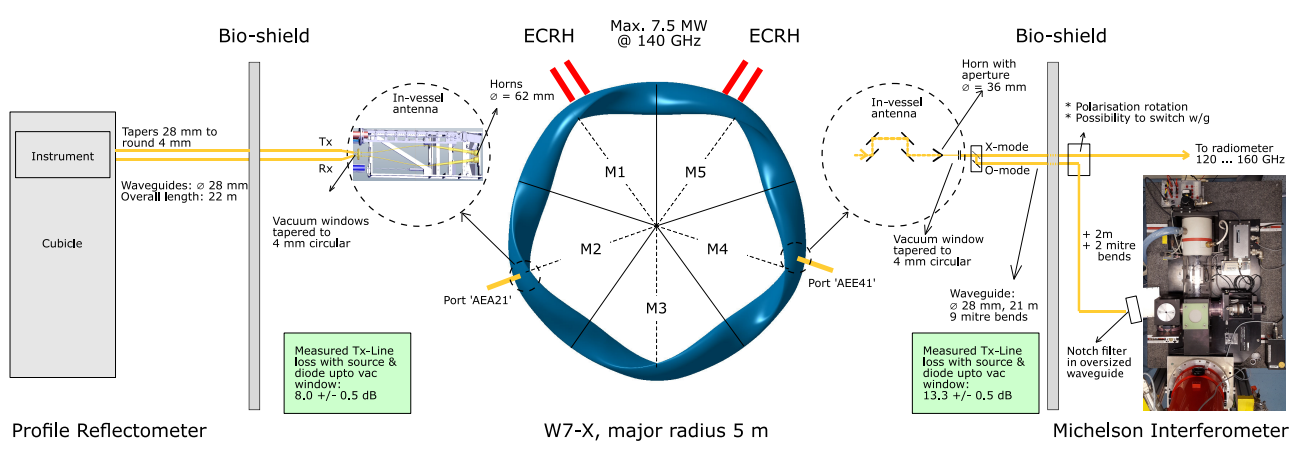

Figure 1: Michelson and Profile Reflectometer waveguide systems at W7-X

\subsection{Stray radiation probe}

The stray radiation measurements were carried out close to the entrance of the instruments, i.e. directly behind the tapers from circular $28 \mathrm{~mm}$ diameter to fundamental. For the stray radiation measurements the $28 \mathrm{~mm}$ circular waveguide was tapered to D-band $(110 \cdots 170 \mathrm{GHz})$. The signal was - by means of a variable attenuator - terminated on a single mode Schottky detector diode. The detectors were calibrated with a $140 \mathrm{GHz}$ source using an isolator and a variable attenuator. With the attenuator set to $0 \mathrm{~dB}$, the output power was measured as $0.6 \mathrm{~mW}$, corresponding to $10 \mathrm{~V}$ at the ADC. The combination of detector diode and pre-amplifier resulted in a sensitivity of the sniffer probe of $17 \mathrm{~V} / \mathrm{mW}$ at low power. The lowest power level that could be detected without additional video conditioning was around $-35 \mathrm{~dB}$ below $0.6 \mathrm{~mW}$ (i.e. $0.2 \mu \mathrm{W}$ ). In the Michelson configuration the probe measured O-mode and in the Profile Reflectometer configuration the probe measured X-mode.

\section{Expected power levels}

\subsection{ECH}

In-vessel stray radiation levels are obtained from [3]. For over-dense plasmas (O2-heating) stray radiation levels as measured by bolometers are of the order of $10 \mathrm{kWm}^{-2}$ for module 2 (Reflectometer) and $4 \mathrm{kWm}^{-2}$ for module 4 (Michelson). This data is for injection of $5 \mathrm{MW} \mathrm{ECH}$. For X2 heated plasmas, optically thick and not in cutoff, stray radiation will predominantly originate from the $\mathrm{ECH}$ launchers and mismatches in polarisation launch, and these levels will be lower. In the presence of a plasma a more or less isotropic and homogeneous power density is found in the volume between the plasma surface and the vessel components. In such case, the stray radiation is present under all angles and polarisations and the absolute ECH power coupled into the horn is obtained by multiplication of the physical antenna aperture with the power density but reduced by the antenna directivity. In this assessment the antenna directivity of the horns is taken to be 20 $\mathrm{dB}$, compared to the ideal situation of an omni-directional sniffer probe at $0 \mathrm{~dB}$. The power that reaches the detector is subsequently reduced by i) rejection of modes, and ii) attenuation of the waveguide for stray radiation. The reduction of modes is here taken to be the ratio of all modes that can propagate to those reaching the detector, i.e. one mode in this case (single mode detector). An estimate considering all possible modes in circular oversized waveguide is given by: $N=0.85\left(\frac{f}{f_{0}}\right)^{2}$ [4], with $f_{0}$ the fundamental cut-off frequency in the waveguide (here $\mathrm{TE}_{11}$ ). For the Michelson horn antenna one finds of the order of 700 modes while for the Reflectometer horn one finds of the order of 2000 modes. Expressed in decibels the ratios multi-mode at horn aperture to single mode at detector are $-28 \mathrm{~dB}$ resp. $-33 \mathrm{~dB}$. The attenuation of the waveguide for stray radiation is here taken to be the same as that measured with the RF-source and diode in section 2 .

\subsection{ECE}

The ECE power is emitted in two modes (X-and O-mode). The circular in-vessel antenna has been arranged such that the antenna pattern is completely illuminated by the ECE in either of these two modes. Under such condition, and with the plasma optically thick, the power coupled into the waveguide is $\approx$ kTB per mode using the Rayleigh-Jeans approximation for black body radiation, with $\mathrm{k}$ Boltzmann's constant, $T$ the electron Temperature and $B$ the bandwidth [5]. The TRAVIS code [6] was used to verify the ECE spectrum for typical temperatures $(5 \mathrm{keV})$ and densities $\left(5 \cdot 10^{19} \mathrm{~m}^{-3}\right)$, see Fig. 2 . It is seen that the power in the 2-nd harmonic O-mode is less the that in the 2-nd harmonic X-mode. As the Michelson probe measures in O2-mode, less power then based on kTB may be expected. When calculating the power over the entire spectrum, one should take the particular electron temperature profile into account as the central electron temperature would result in an over-estimate. In this estimate a parabolic electron temperature profile has been assumed which results in a reduction of the overall power by a factor of 1.5 with respect to a 'top-hat' profile.

\subsection{Estimate}

Table 1 gives the expected power as found using the data in sections 3.1 and 3.2. 

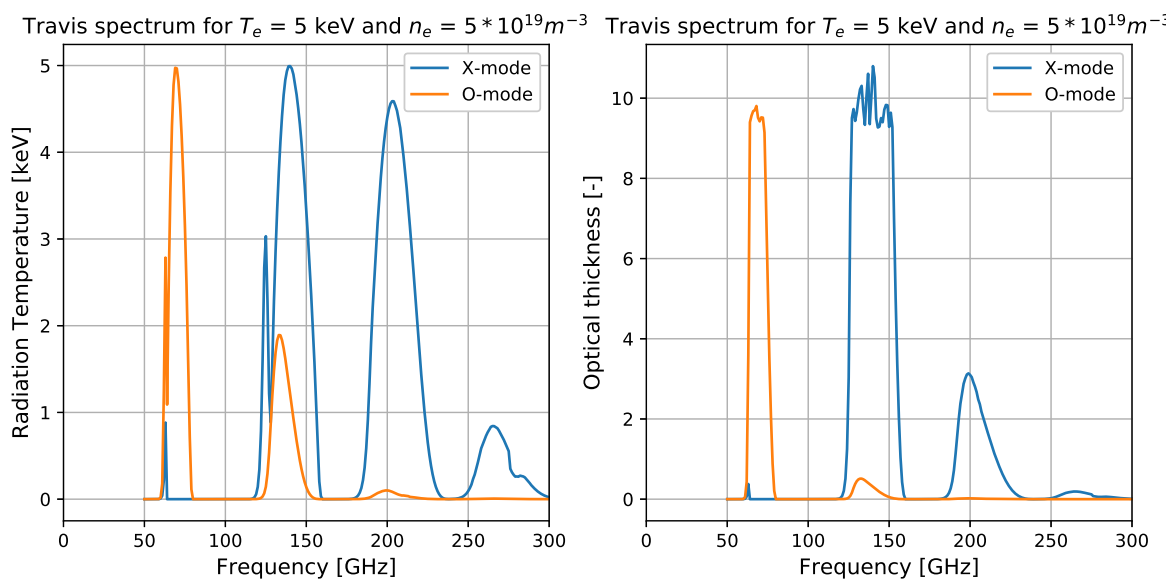

Figure 2: Data from Travis, left: radiation spectrum and right: optical depth.

\begin{tabular}{|l|c|c|c|c|c|c|c|}
\hline & $\begin{array}{c}\text { Power Density } \\
{\left[\mathbf{W m}^{-2}\right] / 5 \mathrm{MW}}\end{array}$ & System & $\begin{array}{c}\text { Ant. dia. } \\
{[\mathrm{mm}]}\end{array}$ & $\begin{array}{c}\mathbf{P}_{\mathbf{2}} \text { ant. } \\
{[\mathrm{W}]}\end{array}$ & $\begin{array}{c}\mathbf{P}_{\text {EEE }}(\mathbf{k T B}) \\
{[\mu \mathrm{W}]}\end{array}$ & $\begin{array}{c}\text { ECH/ECE } \\
@ \text { Ant. [dB] }]\end{array}$ & $\begin{array}{c}\text { ECH/ECE } \\
\text { on Det. [dB] }\end{array}$ \\
\hline Module 2 & 12000 & $\mathrm{PR}$ & 61 & 0.35 & 32 & 40 & 7 \\
\hline Module 4 & 4000 & $\mathrm{Ml}$ & 36 & 0.04 & 32 & 31 & 3 \\
\hline
\end{tabular}

Table 1: Expected power levels. The column $P_{\text {ant }}$ gives the maximum ECH power coupled into the antennae: the power density multiplied with the antenna aperture but reduced by the antenna directivity. PR stands for Profile Reflectometer and MI for Michelson. The column $\mathrm{P}_{E C E}$ is the ECE power over D-Band, taking a central electron temperature of $5 \mathrm{keV}$ and assuming a parabolic profile. Next the power ratio ECH to ECE in-vessel is calculated. The last column gives this ratio in front of the Michelson: here the stray radiation is strongly reduced due to the transition from oversized horn antenna to single mode detector.

\section{Experiment}

Availability of waveguides - in demand for other diagnostic data - restricted the measurements to a number of days towards the end of campaign. Nevertheless useful data was obtained but in X2 heated plasmas only. It was found that RF-attenuation before the diode could be reduced to below a factor 10 before the ECH signal during start-up approached $1 \mathrm{~mW}$ on the detector. During the plasma phase the stray radiation power was mostly too low to detect but note that only X2-heated plasmas were available. Fig. 3 shows a panel with time traces, the left hand column shows the overall pulse, the right hand column a zoom during start-up. The first row ( $\mathrm{a} \& \mathrm{~b}$ ) shows signals for the total ECRH power, the second row (c \& d) the Michelson stray radiation probe ('MI-probe'), and the third row (e $\&$ f) the Profile Reflectometer stray radiation probe ('PRprobe'). In this discharge the central electron temperature was around $5 \mathrm{keV}$. The central density was varied, but between 1 to 4 seconds it was in the range of $5 \cdot 10^{19}$ electrons $\mathrm{m}^{-3}$. The attenuation in front of the MI-probe was $4+/-1$ $\mathrm{dB}$ while for the PR-probe it was set to $0 \mathrm{~dB}$. It is seen that the stray radiation probes, on the $10 \mathrm{~V}$ scale of the ADC, only show signal during the plasma start-up, i.e. when ECRH power is injected but there is no absorption by the plasma yet.

The experimental program was not dedicated to stray radiation measurements. However, polarisation scans with the probes themselves were carried out and the W7-X program required various ECH launcher angles. An initial assessment shows no large deviation from the presented data, however, a systematic analysis of launcher angles and polarisation - requiring additional measurements - is planned.

During start-up the data shows a large standing wave pattern of stray radiation that varied with launching angle and polarisation. But the amplitude rarely clipped the limit of the ADC, and if it did so, only just. During the plasma phase, limited stray radiation (below the levels at start-up) is observed during a number of pulses where the density is too high or too low.

The relatively low waveguide attenuation for the PRprobe, the $0 \mathrm{~dB}$ setting of the attenuator and possibly the $\mathrm{X}$-mode measurement opposed to $\mathrm{O}$-mode, made it possibly to see a finger-print of the ECE during the plasmaphase of the discharge on the PR-probe. This is shown in Fig. 4, now with a vertical zoom on the PR-probe and as a reference a central radiometer EC-channel (\# 14, 138.085 ‥ 138.435 GHz).

\section{Discussion}

Both probes show strong ECH stray radiation during plasma start-up. As the power fluctuates wildly, and is made up out of standing waves, it is not possible to give an accurate estimate of the power. But the signal very rarely 

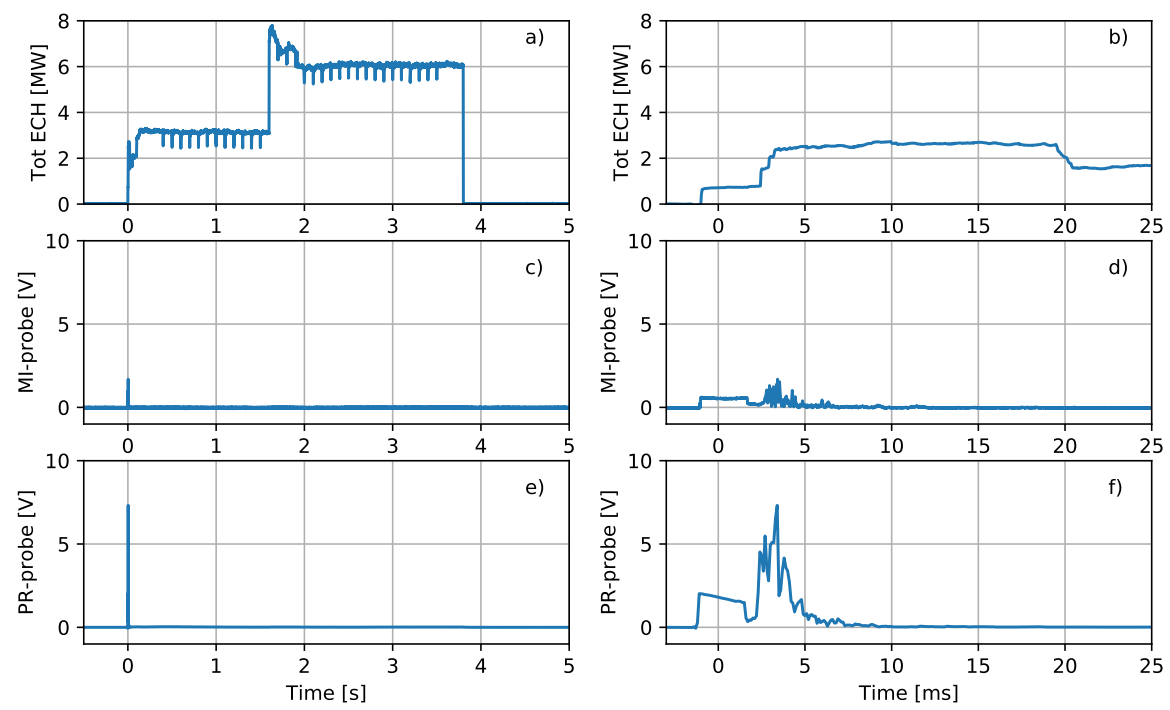

Figure 3: Data for \# 171207009. Traces in the left hand column show the overall discharge, traces in the right hand column a zoom on plasma start-up. Top: ECRH total power, center: the Profile Reflectometer stray radiation probe and lower the Michelson Interferometer stray radiation probe.

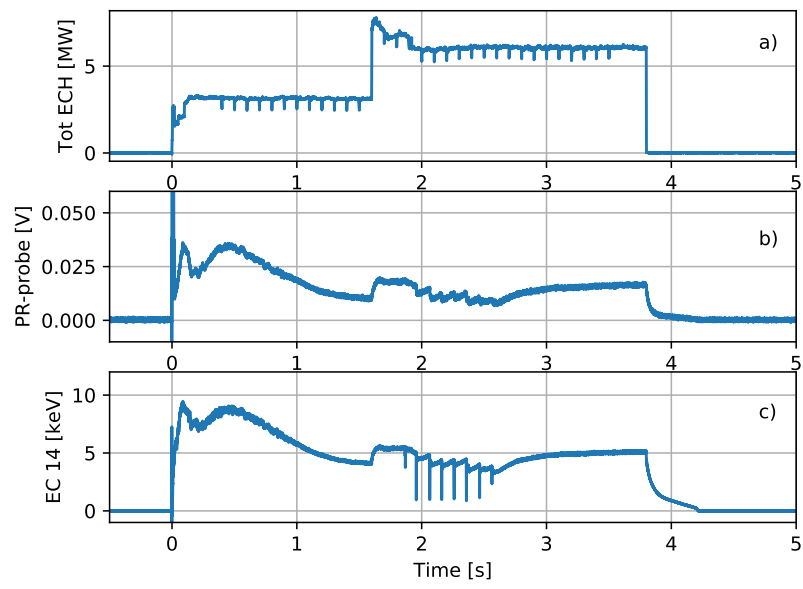

Figure 4: ECE finger-print on Profile Reflectometer stray radiation probe (b) using the data as in Fig. 3. For comparison a central ECE channel is shown (c). After ECH switch-off (@ $3.8 \mathrm{~s}$ ) the signal from the probe falls off exponentially with the confinement time showing that no large ECH stray radiation power component seemed present.

saturated and is limited to $1 \mathrm{~mW}$ in the single mode measured. The Michelson is multi-mode but with the number of modes limited by the $\approx 10$ modes that the $4 \mathrm{~mm}$ section of transmission line in the vacuum window can support (see Sec. 3.1). The total power reaching the Michelson is then expected to be at maximum 10 times larger, i.e. at maximum $10 \mathrm{~mW}$ during start-up. Note that this is based on the estimates of modes, and the assumption that the total power is spread equally over these modes. As the power at start-up is expected (and observed) to be far in excess of stray radiation during the plasma phase of the experiment, the notch filter maximum ECH power is set at $10 \mathrm{~mW}$. The ECE losses from vessel to Michelson are $16 \mathrm{~dB}$, or $\approx$ a factor 40. If one demands the $\mathrm{ECH}$ power to be reduced to the overall ECE power at the input of the Michelson one finds a ratio of $10 \mathrm{~mW} / 1 \mu \mathrm{W}=40 \mathrm{~dB}$. Such a ratio is useful as the detector will remain in the same dynamic range during start-up and during the plasma phase.

During the plasma phase the expected ratio $\mathrm{ECH}$ to overal ECE for a single mode detector is given in the last column of Table 1. For the Michelson, exposed to 10 modes, the ratio is $10 \mathrm{~dB}$ higher. When rounded the ECH power in $\mathrm{O} 2$-heated plasma is expected $\approx 15 \mathrm{~dB}$ more then the overall ECE power. In the experiment, but X2 mode heating opposed to O2-mode, the ratio ECH to overall ECE appears to be below unity.

To validate the ECE component a consistency check can be made. Referring to Fig. 4 the ECE power scales to around $20 \mathrm{mV}$ in the ADC. This signal is the ECE response over $120 \cdots 170 \mathrm{GHz}$ in X-mode (Reflectometer probe) and as such represent a 'spatially averaged' ECE power. From the detector scaling of $17 \mathrm{~V} / \mathrm{mW}$ it follows that $20 \mathrm{mV}$ measured with the ADC corresponds to $\approx$ $1.2 \mu \mathrm{W}$ on the detector. Applying the overall transmission line attenuation of $16 \mathrm{~dB}$ between the instrument and the in-vessel antenna, the power at the in-vessel antenna is 16 $\mathrm{dB}$ more, or $48 \mu \mathrm{W}$. In Table 1 it is estimated to be $\approx 32$ $\mu \mathrm{W}$. . From this it is concluded that the expected $(\approx 30$ $\mu W)$ and measured ECE power $(\approx 50 \mu W)$ are consistent given the margins on such an ECE power measurement. 
The probe on the Michelson does not show an ECE signal. But the losses in the Michelson transmission line are higher by $6+4$ (attenuator) $=10 \mathrm{~dB}$. This reduces the $1.2 \mu \mathrm{W}$ as measured by the Profile Reflectometer probe to $0.12 \mu \mathrm{W}$, indeed at the limit of the probe.

\section{Conclusions and future work}

Measurement complemented by modeling suggest that the maximum stray radiation power during ECH start-up, at the input of the Michelson, will not exceed $10 \mathrm{~mW}$ in the specific W7-X configuration. A notch at $40 \mathrm{~dB}$ brings the signal in the same order of magnitude as the overall expected ECE power. Expected stray radiation levels during the plasma phase in O2-heated plasmas are of the order of $15 \mathrm{~dB}$ above the ECE levels. A notch filter at $40 \mathrm{~dB}$ would cope with this. This margin is comportable given uncertainties such as i) extrapolation from power measured with a single mode detector to power measured in multi-mode with the Michelson, ii) assumtions on the difference in directivity of a sniffer probe compared to a ECE-horn, and iii) a limited data set in ECH heated plasmas as of yet, and excluding O2-heated data. A careful measurement campaing with the Michelson, with the notch filter in place, is required to verify the results.

The authors are grateful for the discussions with the W7-X ECH team, Dr. Walter Kasparek and Prof. Manfred Thumm during this work.
This work has been carried out within the framework of the EUROfusion Consortium and has received funding from the Euratom research and training programme 2014-2018 under grant agreement No 633053. The views and opinions expressed herein do not necessarily reflect those of the European Commission.

\section{References}

[1] M. Moseev et al., Review of Scientific Instruments, 87(8), 1-9. [083505]

[2] S. Marsen et al., Nuclear Fusion, Volume 57, Number 8

[3] S. Marsen, Poster Session during the 20th Joint Workshop on Electron Cyclotron Emission (ECE) and Electron Cyclotron Resonance Heating (ECRH), May 1417, 2018, Greifswald, Germany.

[4] Nikitin et al., Proceedings of the 2011 IEEE International Symposium on Antennas and Propagation (APSURSI), 3-8 July 2011, Spokane, WA, USA

[5] Hartfuss, Hans-Jürgen, Fusion Plasma Diagnostics with mm-Waves (Wiley-VCH, 2013)

[6] N.B. Marushchenko, Computer Physics Communications 185 (2014) 165-176

[7] N. Chaudhary et al., Proceedings of the 20th Joint Workshop on Electron Cyclotron Emission (ECE) and Electron Cyclotron Resonance Heating (ECRH), May 14-17, 2018, Greifswald, Germany. 